\title{
Non-Abelian clouds around Reissner-Nordström black holes: The existence line
}

\author{
Eugen Radu, ${ }^{1}$ D. H. Tchrakian, ${ }^{2,3}$ and Yisong Yang ${ }^{4}$ \\ ${ }^{1}$ Departamento de Fisica da Universidade de Aveiro and CIDMA, \\ Campus de Santiago, 3810-183 Aveiro, Portugal \\ ${ }^{2}$ School of Theoretical Physics-DIAS, 10 Burlington Road, Dublin 4, Ireland \\ ${ }^{3}$ Department of Computer Science, National University of Ireland Maynooth, Maynooth, Ireland \\ ${ }^{4}$ Department of Mathematics, Tandon School, New York University, Brooklyn, New York 11201, USA
}

(Received 19 April 2016; published 27 June 2016)

\begin{abstract}
A known feature of electrically charged Reissner-Nordström-anti-de Sitter planar black holes is that they can become unstable when considered as solutions of Einstein-Yang-Mills theory. The mechanism for this is that the linearized Yang-Mills equations in the background of the Reissner-Nordström (RN) black holes possess a normalizable zero mode, resulting in non-Abelian (nA) magnetic clouds near the horizon. In this work we show that the same pattern may occur also for asymptotically flat RN black holes. Different from the anti-de Sitter case, in the Minkowskian background the prerequisites for the existence of the nA clouds are (i) a large enough gauge group, and (ii) the presence of some extra interaction terms in the matter Lagrangian. To illustrate this mechanism we present two specific examples, one in four- and the other in five-dimensional asymptotically flat spacetime. In the first case, we augment the usual $S U(3)$ Yang-Mills Lagrangian with a higher-order (quartic) curvature term, while for the second one we add the Chern-Simons density to the $S O(6)$ Yang-Mills system. In both cases, an Abelian gauge symmetry is spontaneously broken near a RN black hole horizon with the appearance of a condensate of $\mathrm{nA}$ gauge fields. In addition to these two examples, we review the corresponding picture for anti-de Sitter black holes. All these solutions are studied both analytically and numerically, existence proofs being provided for nA clouds in the background of RN black holes. The proofs use shooting techniques which are suggested by and in turn offer insights for our numerical methods. They indicate that, for a black hole of given mass, appropriate electric charge values are required to ensure the existence of solutions interpolating desired boundary behavior at the horizons and spatial infinity.
\end{abstract}

DOI: 10.1103/PhysRevD.93.124069

\section{INTRODUCTION}

The branching off of a family of solutions of a model into a new family of solutions at the onset of an instability is a recurrent situation in physics. Starting with the vacuum Einstein theory of gravity, an earlier example is the Gregory-Laflamme instability [1] of black strings, which branch off into a family of nonuniform string solutions at the onset of the instability [2]. A more recent example is the set of bumpy black holes (BHs), which branch off the family of Myers-Perry vacuum BHs in $d \geq 6$ spacetime dimensions $[3,4]$.

The same pattern occurs, however, for some field theory models in a flat spacetime background, the best-known example being perhaps the bisphalerons in the electroweak sector of the Standard Model of particle physics [5,6]. As expected, even more complicated solutions are found when considering gravitating matter fields, with new features introduced by the possible existence of an event horizon. A recent example in this direction is found for a complex massive scalar field in the background of a Kerr BH. As discussed in Refs. [7-9], the Klein-Gordon equation possesses bound-state solutions (i.e. scalar clouds) around
Kerr BHs. This leads to the existence of a family of hairy BHs which branch off from the Kerr metric at the threshold of the super-radiant instability [8] (the existence of these scalar clouds at the nonlinear level has been proven recently in Ref. [10]).

An important example which has recently received a considerable amount of interest concerns the instability of the (electrically charged) Reissner-Nordström (RN) BH with a negative cosmological constant when considered as a solution of the gravitating $U(1)$-gauged complex scalar field theory [11], or of the Einstein-Yang-Mills (EYM) theory [12]. In both cases, the branching towards a set of solutions in the more general theory occurs for a particular set of Reissner-Nordström-anti-de Sitter (RNAdS) BHs which form a line in the parameter space. The hairy BHs are the nonlinear realization of those marginally stable modes. This has led to the discovery of a remarkable connection between condensed matter and gravitational physics, the hairy black hole solutions in the aforementioned theories being interpreted as holographic superconductors, via the gauge/gravity duality [13].

The main purpose of the present work is to show that the mechanism resulting in the spontaneous breaking of an 
Abelian gauge symmetry near a black hole horizon, with the appearance of a condensate of a magnetic non-Abelian (nA) gauge field, also occurs for gravitating nA fields in the absence of a cosmological constant. ${ }^{1}$ Following the terminology for scalar fields [7-9], these configurations with infinitesimally small magnetic fields are dubbed here nonAbelian clouds. Moreover, the corresponding bifurcating Reissner-Nordström (RN) BHs will correspond to an existence line in the parameter space of solutions.

There is, however, a price to be paid in this case. First, the gauge group must be larger than $S O(d-1)$ (with $d$ the spacetime dimension), and second, a suitable YM additional interaction term, with its corresponding dimensional constant, must be employed. There are two candidates for this, the nonlinear densities constructed from the nA fields and connections, namely (i) as many higher-order curvature terms as are allowed in the given dimension, and (ii) the allowed Chern-Simons (CS) density in that (odd) dimension.

For economy of presentation, we have chosen to demonstrate this effect for two typical examples, in four and five spacetime dimensions, respectively. In the $d=4$ case, we do not have the option of employing a CS term, and the only higher-order curvature term available there is the quartic kinetic YM term $F^{4}=\operatorname{Tr}\left\{F_{\mu \nu \rho \sigma} F^{\mu \nu \rho \sigma}\right\}$. As for gauge group, we have chosen $S U(3)$, which is the smallest group larger than $S O(4)$ or $S U(2)$. In five dimensions, however, where we have available the CS density, we have eschewed the use of the $F^{4}$ form for simplicity, and have chosen to employ the CS term for diversity. In that case, we have chosen to work with the gauge group $S O(6)$ for convenience.

With these two examples, we aim to illustrate this mechanism for generic cases. Let us also mention that our study uses a combination of analytical and numerical methods, which is enough for most purposes. In particular, we have given analytic proofs of existence in addition to numerical constructions.

The methods of proofs are based on shooting arguments, utilizing the black hole electric charge as a shooting parameter which gives rise to the boundary slope of the gauge field profile function at the horizon. In order to obtain correct values of the charge, two steps of shooting processes are conducted. These methods are hinted at by, and useful to, the numerical approaches employed in this study.

Our work is organized as follows: Before considering the case of main interest of solutions in a Minkowski spacetime background, we start in Sec. II by reviewing the mechanism unveiled in Ref. [12] for the occurrence of nA clouds in the gravitating $S U(2)$ YM system with a negative

\footnotetext{
${ }^{1}$ Note that the (asymptotically flat, magnetically charged) RN $\mathrm{BH}$ is known to be unstable when viewed as a solution of the Einstein-Yang-Mills-Higgs theory [14].
}

cosmological constant. In Secs. III and IV, we discuss two specific examples for the instability of asymptotically flat RN BHs with $\mathrm{nA}$ fields, in four and five dimensions. In the first case, one supplements the usual Yang-Mills Lagrangian with higher-order curvature terms of the gauge field, while for the latter case, one considers the YangMills-Chern-Simons theory. The numerical results there are underpinned by rigorous existence proofs for the solutions of the corresponding linearized (generalized) Yang-Mills equations. We end with Sec. V, where the results are summarized and discussed. The Appendix contains essentially the equations for the full nonlinear systems discussed in Secs. II-IV, put, however, in a more general context.

\section{NON-ABELIAN CLOUDS AROUND $d=4$ REISSNER-NORDSTRÖM-ANTI-DE SITTER BLACK HOLES}

\section{A. The setting}

We consider the usual EYM action supplemented with a cosmological term $\Lambda=-3 / L^{2}$ :

$$
S=\int d^{4} x \sqrt{-g}\left[\frac{1}{4}\left(R+\frac{6}{L^{2}}\right)-\frac{1}{2} \operatorname{Tr}\left\{F^{2}\right\}\right],
$$

where the field-strength tensor $F_{\mu \nu}=\frac{1}{2} \tau^{a} F_{\mu \nu}^{a}$ is $F_{\mu \nu}=$ $\partial_{\mu} A_{\nu}-\partial_{\nu} A_{\mu}+i\left[A_{\mu}, A_{\nu}\right]$, and the gauge field is $A_{\mu}=$ $\frac{1}{2} \tau^{a} A_{\mu}^{a}$, with $\tau^{a}$ an $S U(2)$ basis written in terms of Pauli matrices.

The RNAdS BH with a planar horizon is a solution of this model and has a line element

$$
\begin{aligned}
d s^{2} & =\frac{d r^{2}}{N(r)}+r^{2}\left(d \theta^{2}+\theta^{2} d \varphi^{2}\right)-N(r) d t^{2}, \quad \text { with } \\
N(r) & =-\frac{2 M}{r}+\frac{Q^{2}}{r^{2}}+\frac{r^{2}}{L^{2}}
\end{aligned}
$$

(with $M, Q$ as the two constants fixing the mass and electric charge of the $\mathrm{BH}$ ) and a purely electric gauge field

$$
A=V(r) \frac{1}{2} \tau_{3} d t, \quad \text { with } \quad V(r)=\left(\frac{Q}{r_{H}}-\frac{Q}{r}\right),
$$

where $r_{H}$ is the largest root of the equation $N\left(r_{H}\right)=0$. In the above relations, $r$ and $t$ are the radial and time coordinates, respectively, while $\theta$ and $\varphi$ are coordinates on the two-plane, with $0 \leq \theta<\infty, 0 \leq \varphi<2 \pi$.

The genuine nA solutions are found when exciting the magnetic degrees of freedom of the $S U(2)$ potential, with $[15,16]$

$$
A=V(r) \frac{1}{2} \tau_{3} d t+\frac{1}{2}\left\{w(r) \tau_{1} d \theta+\left(\tau_{3}+w(r) \tau_{2} \theta\right) d \varphi\right\},
$$


and would describe dyonic nA BHs (the corresponding equations of the model are given in Sec. I of the Appendix). Here we are interested in the case when $w(r)$ is an infinitesimally small function

$$
w(r)=\epsilon W(r),
$$

such that the backreaction induced by the magnetic field on the spacetime geometry (2) can be neglected, and the RNAdS BH remains a solution of the model. Then the function $W(r)$ solves the linearized YM equation

$$
W^{\prime \prime}+\frac{N^{\prime}}{N} W^{\prime}+\frac{\left(\frac{Q}{r_{H}}-\frac{Q}{r}\right)^{2}}{N^{2}} W=0,
$$

where a prime denotes a derivative with respect to the coordinate $r$.

\section{B. The parameter dependence of solutions}

The above equation does not seem to possess a closedform solution. One can construct, however, approximate solutions valid for $r \rightarrow r_{H}$ and $r \rightarrow \infty$, respectively. In the vicinity of the event horizon, the regular solution has

$$
W(r)=b+\frac{L^{4} Q^{2} r_{H}^{2} w_{0}}{4\left(L^{2} Q^{2}-3 r_{H}^{4}\right)^{2}}\left(r-r_{H}\right)^{2}+\cdots
$$

(with $b \neq 0$ arbitrary), the corresponding expression for large $r$ being

$$
W(r)=\frac{J}{r}-\frac{L^{4} Q^{2} J}{6 r_{H}^{2}} \frac{1}{r^{3}}+\cdots .
$$

In what follows, we investigate the existence of a smooth solution connecting these asymptotics. Since Eq. (6) is linear and homogeneous, we may set $b=1$ without loss of generality and look for a solution satisfying $W(\infty)=0$. For this purpose, we rewrite $N(r)$ as

$$
N(r)=\frac{1}{r^{2}}\left(\frac{1}{L^{2}} r^{4}-2 M r+Q^{2}\right) \equiv \frac{1}{r^{2}} f(r), \quad r>0
$$

and locate $r_{H}$ first. It is seen that the only root of $f^{\prime}(r)$ is $r_{0}=2^{-\frac{1}{3}} M^{\frac{1}{3}} L^{\frac{2}{3}}$. In order for $N(r)$ to have a positive root, it is necessary and sufficient to require $f\left(r_{0}\right) \leq 0$, which leads to the condition

$$
Q^{2} \geq 2^{\frac{4}{3}} M^{\frac{4}{3}} L^{\frac{2}{3}}\left(1-2^{-\frac{8}{3}}\right) \equiv Q_{0}^{2}, \quad Q_{0}>0 .
$$

If $Q=Q_{0}, N(r)$ will have a double root at $r=r_{0}$; if $Q>Q_{0}, N(r)$ will have two positive simple roots which both depend on $Q$ and may be denoted as $r_{1}(Q)$ and $r_{2}(Q)$, with $r_{1}(Q)<r_{2}(Q)$. Thus, in our notation $r_{H}=r_{2}(Q)$. Since $f(r)>-2 M r+Q^{2}$, we have

$$
\frac{Q^{2}}{2 M}<r_{1}(Q)<r_{2}(Q)=r_{H}(Q) \text {. }
$$

Suggested by the asymptotic expression (7), we are to obtain a positive solution of (6) subject to the boundary condition

$$
W\left(r_{H}\right)=1, \quad W(\infty)=0,
$$

following from (7) and (8). With this, we may begin by integrating (6) to represent the unique local solution with the initial condition $W\left(r_{H}\right)=1, W^{\prime}\left(r_{H}\right)=0$ as

$W^{\prime}(r)=-\frac{1}{N(r)} \int_{r_{H}}^{r} \frac{Q^{2}}{N(\rho) r_{H}^{2} \rho^{2}}\left(\rho-r_{H}\right)^{2} W(\rho) d \rho, \quad r>r_{H}$.

Integrating (11), we formally arrive at

$$
\begin{aligned}
W(r) & =1-\int_{r_{H}}^{r} \frac{1}{N(s)} \int_{r_{H}}^{s} \frac{Q^{2}}{N(\rho) r_{H}^{2} \rho^{2}}\left(\rho-r_{H}\right)^{2} W(\rho) d \rho d s, \\
r & >r_{H} .
\end{aligned}
$$

The parameter dependence of the solution is demonstrated in (11) and (12), which allows us to obtain desired solution profiles by parameter adjustment.

\section{The numerical results}

In practice, the solution interpolating between the asymptotics (7) and (8) is found numerically, a typical profile being shown in Fig. 1. Note that we restrict here to configurations with $W(r)$ everywhere positive; however, there are also excited solutions in which $W(r)$ has nodes [12].

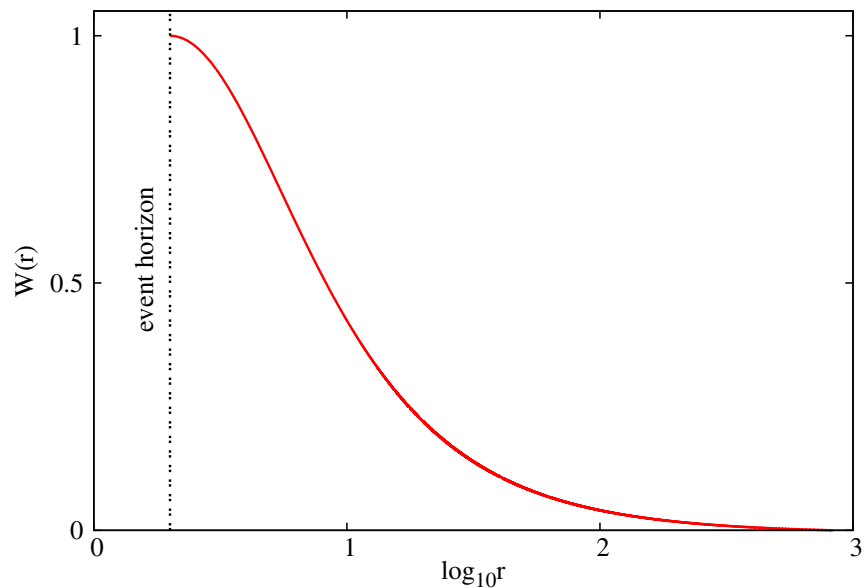

FIG. 1. The profile of a typical solution of Eq. (6). The parameters of the corresponding RNAdS background are $M=0.0453, Q=0.1465$, and $L=10$. 


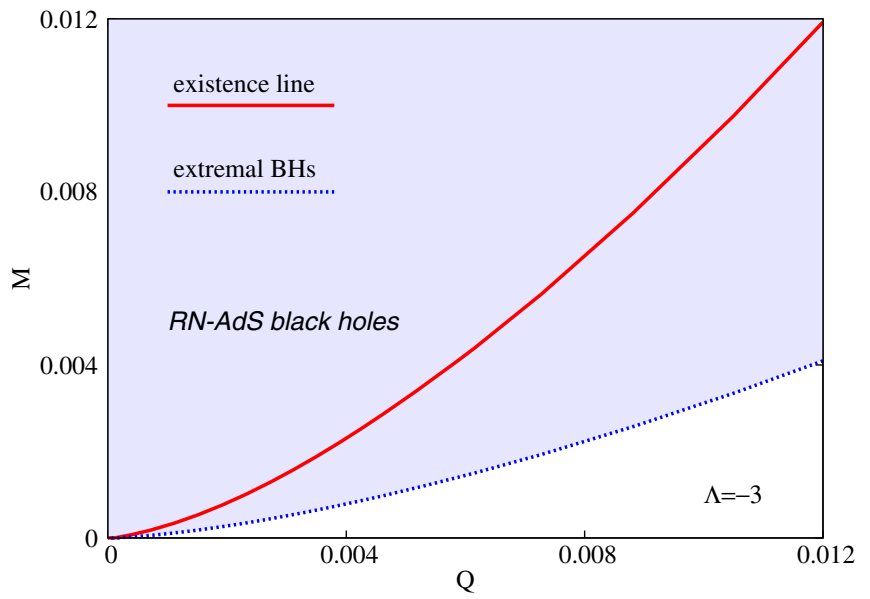

FIG. 2. Mass $M$ vs charge $Q$ for RNAdS black holes in $d=4$ dimensions. The dotted blue curve corresponds to extremal BHs [RN BHs exist above it (shaded region)]. The non-Abelian clouds exist along the red line.

The results of the numerical integration are shown in Fig. 2, where we exhibit the existence line in the $(M, Q)$ parameter space of RNAdS solutions (note that there we set $L=1$ ). One can see that, given a value of the electric charge $Q$, the (nodeless) solution of (6) is found for a critical RNAdS BH only.

As discussed in Ref. [12], when taking into account the backreaction at the nonlinear level, this results in the occurrence of a branch of EYM hairy black hole solutions which bifurcate from the RNAdS configurations precisely at the existence line. Moreover, the solutions with a nonzero magnetic potential are thermodynamically favored over the magnetically neutral ones (i.e., they maximize the entropy for given $M, Q$ ).

\section{NON-ABELIAN CLOUDS AROUND $d=4$ REISSNER-NORDSTRÖM BLACK HOLES WITH MINKOWSKI SPACETIME ASYMPTOTICS}

\section{A. The setting}

It is worth inquiring to which extent the features unveiled above are specific to AdS spacetime, and whether they can be recovered (at least to some extent) also by asymptotically flat configurations. The first observation is that in this case the BHs necessarily possess a spherical horizon topology $[17,18]$. Second, for $\Lambda=0$, the static configurations with a gauge group $S U(2)$ and a nontrivial magnetic potential necessarily have $A_{t} \equiv 0$ as found by a number of classic no-go "baldness" theorems [19]. This immediately excludes the existence of $S U(2)$ clouds around electrically charged Reissner-Nordström black holes.

One may hope that the situation changes when considering instead a larger gauge group. The minimal gauge group for which the superposition of a Coulomb field and non-Abelian hair is not forbidden by the "baldness" theorems is $S U(3)$. In this case, a spherically symmetric ansatz for the gauge field reads ${ }^{2}$ [20]

$A=w(r) T_{1} d \theta+\left(w(r) T_{2} \sin \theta+\cos \theta T_{3}\right) d \varphi+V(r) T_{8} d t$,

where $T_{i}$ are the standard generators of the $S U(3)$ Lie algebra. The electrically charged $\mathrm{RN} B \mathrm{BH}$ remains a solution of the EYM-SU(3) model, with a line element

$$
\begin{aligned}
d s^{2} & =\frac{d r^{2}}{N(r)}+r^{2}\left(d \theta^{2}+\sin ^{2} \theta^{2} d \varphi^{2}\right)-N(r) d t^{2}, \quad \text { with } \\
N(r) & =1-\frac{2 M}{r}+\frac{Q^{2}}{r^{2}},
\end{aligned}
$$

(with $M$ and $Q$ the mass and electric charge of $\mathrm{BHs}$ ) and an embedded Abelian connection

$$
w(r)= \pm 1, \quad V(r)=\left(\frac{Q}{r_{H}}-\frac{Q}{r}\right)
$$

where $r_{H}=M+\sqrt{M^{2}-Q^{2}}>0$ is an input parameterthe outer event horizon [with $N\left(r_{H}\right)=0$ ].

Apart from that, there are also genuinely nA solutions which possess a nontrivial magnetic potential $w(r)$. As discussed in Ref. [20], these configurations can be thought of as nonlinear superpositions of the (electric) RN and the (purely magnetic) $S U(2)$ black holes [21]. In particular, they do not emerge as perturbations of the electrically charged BHs, the overall picture being very different from that in the AdS case.

These results are found for a usual YM action which contains the usual quadratic term $F_{\mu \nu} F^{\mu \nu}$ only. However, as discussed in Ref. [22], the situation changes when the nA action is augmented with higher-order curvature terms of the gauge field. In the simplest case, the Lagrangian for the $S U(3)$ fields reads

$$
\begin{aligned}
\mathcal{L} & =-\frac{1}{2} \operatorname{Tr}\left\{F_{\mu \nu} F^{\mu \nu}\right\}+\mathcal{L}_{s}, \quad \text { with } \\
\mathcal{L}_{s} & =\frac{3 \tau}{2} \operatorname{Tr}\left\{\left(F_{\mu \nu} \tilde{F}^{\mu \nu}\right)^{2}\right\}
\end{aligned}
$$

where a tilde denotes the Hodge dual and $\tau$ is an input parameter of the theory. One can see that $\mathcal{L}_{s}$ features only the second power of any "velocity field" and is a causal density just like the Gauss-Bonnet term in gravity [23] or the Skyrme [24] term of the $O(4)$ sigma model. Also, $\mathcal{L}_{s}$ can be viewed as the second member of the YM hierarchy [25], providing a natural generalization of the usual YM

\footnotetext{
${ }^{2}$ Note that Eq. (13) corresponds in fact to an $S U(2) \times U(1)$ ansatz, the magnetic and electric potential interacting only via the spacetime geometry.
} 
model. Such terms were extensively considered in the literature for Abelian solutions; however, the $\mathrm{nA}$ case is considerably less studied.

The equations of motion for a spherically symmetric system are displayed in Sec. II of the Appendix. An important observation is that the (electrically charged) $\mathrm{RN} \mathrm{BH}$ is still a solution of this model with the extra $\mathcal{L}_{s}$ term. We are interested in the configurations with $w^{2}(r)$ infinitesimally close to 1 , such that the magnetic part of the $\mathrm{nA}$ field is too small to backreact significantly upon the geometry,

$$
w(r)=-1+\epsilon W(r),
$$

in which case the geometry is still described by the metric functions in (14), sourced by a purely electric YM field.

Then the linearized YM equations in Sec. II of the Appendix imply that $W(r)$ solves the equation

$$
\left(N W^{\prime}\right)^{\prime}=\frac{2 W}{r^{2}}\left(1-\frac{2 Q^{2} \tau}{r^{4}}\right)
$$

\section{B. The existence of solutions}

We are interested in the smooth solutions of (18) with the following form as $r \rightarrow r_{H}$ :

$$
W(r)=b+w_{1}\left(r-r_{H}\right)+O\left(r-r_{H}\right)^{2},
$$

where

$$
w_{1}=\frac{2 b\left(r_{H}^{4}-2 Q^{2} \tau\right)}{r_{H}^{3}\left(r_{H}^{2}-Q^{2}\right)}
$$

with $b \neq 0$ an arbitrary parameter. Also, since Eq. (18) is linear, we set $b=1$ without any loss of generality. The corresponding expression for large $r$ reads

$$
W(r)=\frac{J}{r}+\frac{3 J\left(Q^{2}+r_{H}^{2}\right)}{4 r_{H}} \frac{1}{r^{2}}+O\left(1 / r^{3}\right),
$$

with $J$ a constant.

The existence of a smooth solution interpolating between these asymptotics can be shown as follows. First, we fix the length scale of the problem by taking $r_{H}=1$, such that

$N(r)=\left(1-\frac{1}{r}\right)\left(1-\frac{Q^{2}}{r}\right), \quad 0<Q<1, \quad r \geq 1$.

From (19) and (20), the solutions of (18) are subject to the boundary condition

$$
W(1)=1, \quad W(\infty)=0 .
$$

It is easy to see that (18) has a unique local solution satisfying the initial condition

$$
W(1)=1, \quad W^{\prime}(1)=a, \quad a \in \mathbb{R} .
$$

For convenience, we denote such a solution as $W(r ; a)$ and define the set

$S^{+}=\left\{a \in \mathbb{R} \mid W^{\prime}(r ; a)=W_{r}(r ; a)>0\right.$ for some $\left.r>1\right\}$.

It is clear that $S^{+}$is open. It is trivial that $(0, \infty) \subset S^{+}$.

We recast (18) into

$$
\begin{aligned}
W^{\prime}(r ; a) & =\frac{1}{(r-1)} \frac{r^{2}}{\left(r-Q^{2}\right)} \int_{1}^{r} \frac{2}{\rho^{2}}\left(1-\frac{2 Q^{2} \tau}{\rho^{4}}\right) W(\rho ; a) d \rho, \\
r & >1 .
\end{aligned}
$$

Letting $r \rightarrow 1$ in (24) and applying l'Hôpital's rule, we find

$$
a=\lim _{r \rightarrow 1} W^{\prime}(r ; a)=\frac{2\left(1-2 Q^{2} \tau\right)}{\left(1-Q^{2}\right)} .
$$

So we may adjust $Q$ in the interval $(0,1)$ to make $a>0$ or $a \in S^{+}$.

In order to have a solution with $a<0$, we need to request

$$
2 Q^{2} \tau>1
$$

which will be observed in the sequel.

We next consider the interval

$$
I_{0}=\left[1, r_{0}\right] \equiv\left[1,\left(2 Q^{2} \tau\right)^{\frac{1}{4}}\right]
$$

From (24) we see that $W^{\prime}<0$, provided that $r \in I_{0}$ and $W$ stays non-negative for $r \in I_{0}$. With this condition, we have

$$
\begin{aligned}
W^{\prime}(r ; a) & >\frac{r^{2}}{(r-1)\left(r-Q^{2}\right)} \int_{1}^{r} \frac{2}{\rho^{2}}\left(1-\frac{r_{0}^{4}}{\rho^{4}}\right) d \rho \\
& >\frac{2 r_{0}^{2}}{5\left(r_{0}-Q^{2}\right)}\left(r^{4}-r_{0}^{4}\right), \quad r \in I_{0}=\left[1, r_{0}\right]
\end{aligned}
$$

Integrating the above, we get the lower bound

$$
\begin{aligned}
W(r ; a) & >1+\frac{2 r_{0}^{2}}{5\left(r_{0}-Q^{2}\right)}\left(\frac{1}{5}\left[r^{5}-1\right]-r_{0}^{4}[r-1]\right) \\
& \geq 1+\frac{2 r_{0}^{2}}{5\left(r_{0}-Q^{2}\right)}\left(\frac{1}{5}\left[r_{0}^{5}-1\right]-r_{0}^{4}\left[r_{0}-1\right]\right), \\
r & \in I_{0},
\end{aligned}
$$


which may be made positive by adjusting $Q$ in the interval (26) suitably.

Similarly, we can find $a$ suitably such that $W(r ; a)<0$ for some $r \in\left(1, r_{0}\right)$. We set

$$
\begin{array}{ll}
A^{+}=\{a \mid W(r ; a)>0 & \text { for all } \left.r \in I_{0}\right\}, \\
A^{-}=\{a \mid W(r ; a)<0 & \text { for some } \left.r \in I_{0}\right\} .
\end{array}
$$

Then $A^{+}$and $A^{-}$are open sets which may be restricted to an open interval, say $I$, for $Q$ satisfying the condition (26). The connectedness of $I$ implies $A^{0}=I \backslash\left(A^{+} \cup A^{-}\right) \neq \emptyset$.

Take $a \in A^{0}$. Then $W(r ; a) \geq 0$ for all $r \in I_{0}$ and $W\left(r_{1} ; a\right)=0$ for some $r_{1} \in I_{0}$. It is clear that $r_{1}=r_{0}$; otherwise, $r_{1}$ would be an interior minimum of $W$ over $I_{0}$, resulting in $W^{\prime}\left(r_{1} ; a\right)=0$; thus $W \equiv 0$ by the uniqueness theorem of the initial-value problem of ordinary differential equations, which contradicts the initial condition $W(1 ; a)=1$. Thus, we have

$$
W(r ; a)>0 \quad \text { for } r \in\left[1, r_{0}\right), \quad W\left(r_{0} ; a\right)=0 .
$$

Inserting (30) into (24), we obtain

$$
W^{\prime}(r ; a)<0, \quad r \in I_{0} .
$$

We claim $W^{\prime}(r ; a)<0$ for all $r>1$. In fact, for $r$ near and above $r_{0}$, we have from (24)

$$
\begin{aligned}
W^{\prime}(r ; a)= & \frac{1}{(r-1)} \frac{r^{2}}{\left(r-Q^{2}\right)}\left(\int_{1}^{r_{0}}+\int_{r_{0}}^{r}\right) \\
& \times\left(\frac{2}{\rho^{2}}\left[1-\frac{2 Q^{2} \tau}{\rho^{4}}\right] W(\rho ; a)\right) d \rho,
\end{aligned}
$$

which will continue to stay negative for all $r>r_{0}$, since $W$ becomes negative beyond $r_{0}$.

Therefore, we have shown that the set

$$
S^{-}=\left\{a \in \mathbb{R} \mid W^{\prime}(r ; a)<0 \text { for all } r>1\right. \text { and }
$$

$W(r ; a)<0 \quad$ for some $r>1\}$

is not empty. This set is clearly open.

Using the connectedness of $\mathbb{R}$, we see that $S^{0}=\mathbb{R} \backslash\left(S^{+} \cup S^{-}\right) \neq \emptyset$. For $a \in S^{0}$, we have

$$
W^{\prime}(r ; a) \leq 0, \quad W(r ; a) \geq 0, \quad r>1 .
$$

Applying the uniqueness theorem of the initial-value problem of ordinary differential equations again, we get $W>0$.

We claim $W^{\prime}<0$ for all $r>1$. In fact, we have $W^{\prime}(r ; a)<0$ for $r \in I_{0}$, since $W(r ; a)>0$ for $r \in I_{0}$. Assume otherwise that $W^{\prime}$ has at least 1 zero and set

$$
r_{1}=\inf \left\{W^{\prime}(r ; a)=0 \mid r>r_{0}\right\} \text {. }
$$

Then $r_{1}>r_{0}$ and

$$
\begin{aligned}
\int_{1}^{r_{1}}\left(\frac{2}{\rho^{2}}\left[1-\frac{2 Q^{2} \tau}{\rho^{4}}\right] W(\rho ; a)\right) d \rho \\
=W^{\prime}\left(r_{1} ; a\right) \frac{\left(r_{1}-1\right)\left(r_{1}-Q^{2}\right)}{r_{1}^{2}}=0 .
\end{aligned}
$$

Using this result in the decomposition (32) while setting $r_{0}=r_{1}$ and applying $W>0$, we have

$W^{\prime}(r ; a)=\frac{1}{(r-1)} \frac{r^{2}}{\left(r-Q^{2}\right)} \int_{r_{1}}^{r} \frac{2}{\rho^{2}}\left(1-\frac{r_{0}}{\rho^{4}}\right) W(\rho ; a) d \rho>0$,

which is false. Thus, we have strengthened (33) into

$$
W^{\prime}(r ; a)<0, \quad W(r ; a)>0, \quad \forall r>1 .
$$

With the properties of $W$ stated in (35), we now establish the limit

$$
\lim _{r \rightarrow \infty} W(r ; a) \equiv W_{\infty}=0 .
$$

In fact, if $W_{\infty} \neq 0$, then

$$
W(r ; a)>W_{\infty}>0, \quad \forall r>1 .
$$

From (24) we see that

$$
W_{1} \equiv \lim _{r \rightarrow \infty} W^{\prime}(r ; a)
$$

exists. Since (36) exists, we see that $W_{1}=0$ in (38). Hence, by l'Hôpital's rule and (18), we have

$$
\lim _{r \rightarrow \infty} \frac{N W^{\prime}(r ; a)}{\left(-\frac{1}{r}\right)}=\lim _{r \rightarrow \infty} r^{2}\left(N W^{\prime}(r ; a)\right)^{\prime}=2 W_{\infty} .
$$

Since $W_{\infty}>0$, we can find some $r_{2}>0$ sufficiently large so that

$$
\frac{N W^{\prime}}{\left(-\frac{1}{r}\right)}>W_{\infty}, \quad r>r_{2}
$$

That is,

$$
W^{\prime}<N W^{\prime}<-\frac{W_{\infty}}{r}, \quad r>r_{2},
$$

since $N<1$ for $r>r_{0}$. Integrating (40) leads to

$$
W(r ; a)<W\left(r_{2} ; a\right)-W_{\infty} \ln \frac{r}{r_{2}}, \quad r>r_{2},
$$




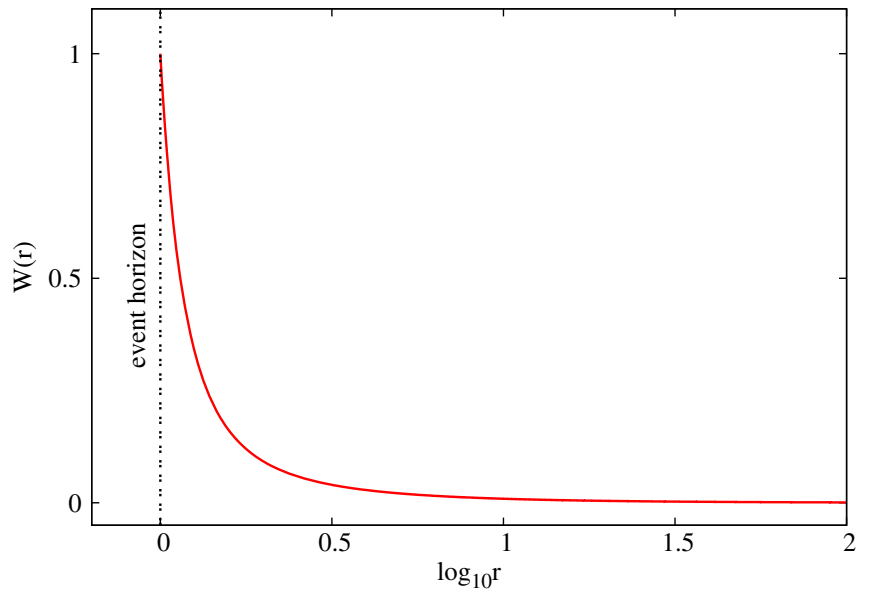

FIG. 3. The profile of a typical solution of Eq. (18). The parameters of the corresponding $\mathrm{RN}$ background are $M=0.8465, Q=0.8324$, and $\tau=1.385$.

so that $W$ fails to stay positive when $r$ is large enough. This contradiction shows that $W_{\infty}$ can only be zero.

In summary, we have established that the initial-value problem consisting of (18) and (23) has a solution $W$ satisfying the boundary condition (22) when (26) is fulfilled and the initial slope parameter $a$ is suitably chosen in the range

$$
a=\frac{2\left(1-2 Q^{2} \tau\right)}{\left(1-Q^{2}\right)}<0
$$

Moreover, $W$ enjoys the properties stated in (35).

\section{The numerical results}

The solutions of (18) interpolating between the asymptotics (19) and (20) are again constructed numerically. In our numerical approach, we fix $r_{H}=1$ and $\tau=1$ as input parameters and look for nodeless solutions. Then (18) becomes an eigenvalue problem in terms of $Q$. The corresponding value of $Q$ is found by using a (numerical) shooting procedure, being uniquely fixed under these assumptions.

The profile of the solution for a typical RN background is shown in Fig. 3. In Fig. 4 we show the existence line of solutions in the $(M, Q)$ parameter space. This corresponds to the critical RN solutions which bifurcate into a branch of BHs with nA hair. The hairy solutions are found when promoting the $\mathrm{nA}$ cloud to a nonlinear level and taking into account the backreaction [22].

\section{HIGHER-DIMENSIONAL GENERALIZATIONS IN MINKOWSKIAN BACKGROUNDS: $d=5$}

\section{A. The setting}

It is of interest to see if the pattern discussed in the previous two sections occurs also in more than four

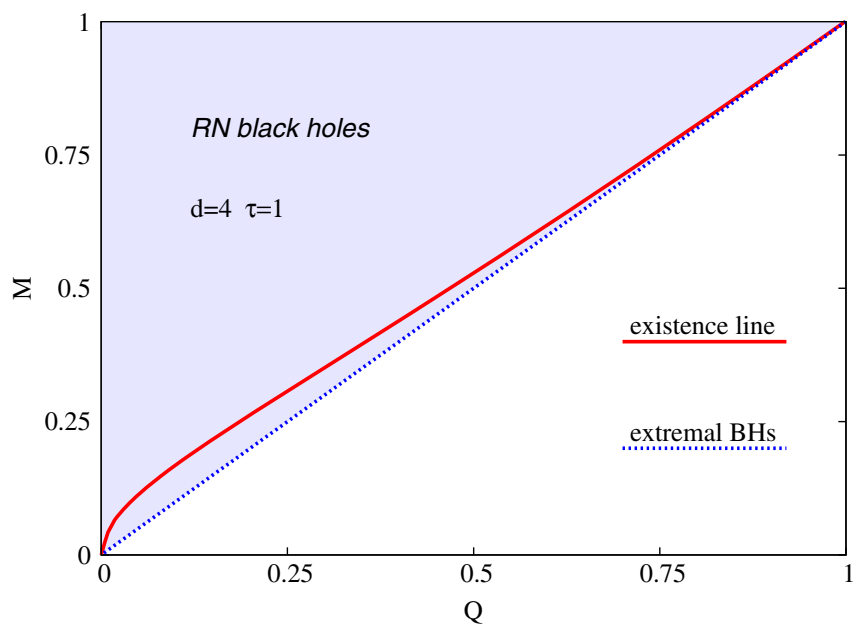

FIG. 4. Mass $M$ vs charge $Q$ for RN black holes in $d=4$ dimensions. The dotted blue curve corresponds to extremal BHs [RN BHs exist above it (shaded region)]. The non-Abelian clouds exist along the red line.

dimensions, $d>4$. As discussed in Ref. [26], the higher-dimensional (planar) RNAdS black holes are also unstable when considered as solutions of EYM theory with a negative cosmological constant. This results as well in the occurrence of a set of critical (embedded Abelian) $\mathrm{BH}$ solutions possessing nA clouds.

We expect that this is also the case for the solutions in a Minkowski spacetime background like that discussed in Sec. III. Indeed, at least in $d=5$ and $d=6$ dimensions, this pattern repeats for the Lagrangian (16) with gauge group $S O(d+1)$, the general setup being presented in Sec. II of the Appendix. By solving the linearized YM equation (A12), we have verified that $\mathrm{nA}$ clouds around RN black holes exist in those cases. The overall picture is similar to that discussed in the previous section, and it will be reported elsewhere [27]. Also, the existence proof given in the previous section can be adapted to the higher-dimensional case.

In what follows, we shall not exercise the option of using higher-order YM terms, but rather will employ a ChernSimons (CS) term in the YM action (16) in lieu of the $F(4)^{2}$ in (16) as the simpler, or less nonlinear alternative. ${ }^{3}$ Moreover, this option has a good justification, since a CS term appears in various supersymmetric theories, the $\mathcal{N}=8, D=5$ gauged supergravity model $[29,30]$ being perhaps the best-known case, due to its role in the conjectured AdS/CFT correspondence.

\footnotetext{
${ }^{3}$ The Chern-Simons terms can be employed in all (higher) odd dimensions, but finite-energy solutions exist only for Lagrangians in which the lowest-order YM term $F(2 p)^{2}$ is of higher order than the $p=2$ case in (16). Of course, the ReissnerNordström solutions in that case would be those of the higherorder gravities studied in Ref. [28]. In that case, one might also replace the usual Einstein gravity with its higher-order versions employed there.
} 
EUGEN RADU, D. H. TCHRAKIAN, and YISONG YANG

In $d=5$ dimensions, the CS Lagrangian reads

$\mathcal{L}_{s}=\kappa \epsilon_{I_{1} \ldots I_{6}}\left(F^{I_{1} I_{2}} \wedge F^{I_{3} I_{4}} \wedge A^{I_{5} I_{6}}-F^{I_{1} I_{2}} \wedge A^{I_{3} I_{4}} \wedge A^{I_{5} J} \wedge A^{J I_{6}}\right.$

$$
\left.+\frac{2}{5} A^{I_{1} I_{2}} \wedge A^{I_{3} J} \wedge A^{J I_{4}} \wedge A^{I_{5} K} \wedge A^{K I_{6}}\right),
$$

where $A^{I J}$ are the $S O(6)$ gauge fields, $F^{I J}=d A^{I J}+$ $A^{I K} \wedge A^{K J}$, and $\kappa$ is the CS coefficient. The gauge field ansatz contains only two functions-a magnetic gauge potential $w$, and a $U(1)$ electric one, $V$ :

$A=\frac{w(r)+1}{r} \gamma_{i j} \frac{x^{i}}{r} d x^{j}+V(r) \gamma_{56} d t, \quad$ with $i, j=1, \ldots, 4$,

with $\gamma_{i j}$ being the representation matrices of $S O(4)$, and $\gamma_{56}$ those of the $S O(2)$, which are subalgebras of $S O(6)$. The Cartesian coordinates $x^{i}$ are related to the spherical coordinates in (45) as in flat space.

The CS term (42) is added to the usual EYM Lagrangian, the resulting equations being displayed in Sec. III of the Appendix. The electrically charged RN BH is a solution of this model, with

$$
w(r) \equiv-1, \quad V(r)=\frac{Q}{r^{2}}-\frac{Q}{r_{H}^{2}},
$$

and a line element

$$
\begin{aligned}
d s^{2} & =\frac{d r^{2}}{N(r)}+r^{2} d \Omega_{3}^{2}-N(r) d t^{2}, \quad \text { with } \\
N(r) & =1-\frac{M}{r^{2}}+\frac{Q^{2}}{r^{4}},
\end{aligned}
$$

where $M, Q$ are two parameters fixing the mass and electric charge of $\mathrm{BH}$, respectively. Also, the outer $\mathrm{BH}$ horizon is located at $r=r_{H}=\left(M+\sqrt{M^{2}-4 Q^{2}}\right) / 2>0$, where $N\left(r_{H}\right)=0$.

Restricting again to an infinitesimally small magnetic field, one takes again

$$
w(r)=-1+\epsilon W(r),
$$

with $W(r)$ a solution of the equation [which results from linearizing the $w$ equation in (A15)]

$$
r\left(r N W^{\prime}\right)^{\prime}=4\left(1+\frac{4 Q \kappa}{r^{2}}\right) W .
$$

\section{B. The existence of solutions}

We are interested in smooth solutions of the above equation which interpolate between the following boundary values:
PHYSICAL REVIEW D 93, 124069 (2016)

$$
W\left(r_{H}\right)=b, \quad W(\infty)=0 .
$$

An approximate solution compatible with (48) can also be constructed; for $r \rightarrow r_{H}$, one finds

$W(r)=b+\frac{2 b r_{H}\left(4 \kappa Q+r_{H}^{2}\right)}{r_{H}^{4}-Q^{2}}\left(r-r_{H}\right)+O\left(r-r_{H}\right)^{2}$,

with $b \neq 0$ an arbitrary parameter. Since Eq. (47) is linear, we set $b=1$ without any loss of generality. The corresponding expression for large $r$ reads

$W(r)=\frac{J}{r^{2}}+\frac{2 J\left(Q^{2}+2 \kappa r_{H}^{2}+r_{H}^{4}\right)}{3 r_{H}^{2}} \frac{1}{r^{4}}+O\left(1 / r^{6}\right)$,

with $J$ a constant.

To simplify notation, we set $r_{H}=1$. Hence,

$N(r)=1-\frac{Q^{2}+1}{r^{2}}+\frac{Q^{2}}{r^{4}}=\frac{1}{r^{4}}\left(r^{2}-1\right)\left(r^{2}-Q^{2}\right)$,

and $Q$ satisfies

$$
-1<Q<0, \quad 1+4 Q \kappa<0,
$$

which may be combined into the compressed condition

$$
-1<Q<-\frac{1}{4 \kappa}, \quad 0<\kappa<\frac{1}{2} .
$$

On the other hand, from (47) we have

$$
\begin{aligned}
W^{\prime}(r) & =\frac{4 r^{3}}{\left(r^{2}-1\right)\left(r^{2}-Q^{2}\right)} \int_{1}^{r} \frac{1}{\rho}\left(1+\frac{4 Q \kappa}{\rho^{2}}\right) W(\rho) d \rho, \\
r & >1 .
\end{aligned}
$$

Using l'Hôpital's rule, we find

$$
b(Q) \equiv W^{\prime}(1)=\lim _{r \rightarrow 1} W^{\prime}(r)=\frac{2}{1-Q^{2}}(1+4 Q \kappa)<0 .
$$

We note that

$$
\lim _{Q \rightarrow-\frac{1}{4 \kappa}} b(Q)=0, \quad \lim _{Q \rightarrow-1} b(Q)=-\infty,
$$

and

$$
b^{\prime}(Q)=\frac{4}{\left(1-Q^{2}\right)^{2}}\left(2 \kappa Q^{2}+Q+2 \kappa\right),
$$

which is positive if $\kappa$ satisfies the additional condition $\kappa>\frac{1}{4}$. Hence, we collect our condition on $\kappa$ here: 


$$
\frac{1}{4}<\kappa<\frac{1}{2} \text {. }
$$

Under this condition, the function $b(Q)$ strictly increases in the interval $\left(-1,-\frac{1}{4 \kappa}\right)$ with values from $-\infty$ to zero. Such a property is essential for the construction of our solution to follow.

In (52), the weight function changes its sign at $\rho=r_{0}$, where

$$
r_{0}=2 \sqrt{-Q \kappa}>1
$$

So we first study the properties of the local solution of (52) in $I_{0}=\left[1, r_{0}\right]$.

We note that $W(r)>0$ when $r>1$ and $r$ is close to 1 . Thus, as long as $W$ stays non-negative and $r \in I_{0}$, we have $W^{\prime}(r)<0$. Thus, in this interval, we have $0 \leq W(r)<1$, and so

$$
\begin{aligned}
W^{\prime}(r) & >\frac{4 r^{3}}{\left(r^{2}-1\right)\left(r^{2}-Q^{2}\right)} \int_{1}^{r} \frac{1}{\rho}\left(1+\frac{4 Q \kappa}{\rho^{2}}\right) d \rho \\
& =\frac{4 r^{3}}{\left(r^{2}-1\right)\left(r^{2}-Q^{2}\right)}\left(\ln r+\frac{r_{0}^{2}}{2}\left[\frac{1}{r^{2}}-1\right]\right) \\
& >\frac{2 r^{3} r_{0}^{2}}{\left(r^{2}-1\right)\left(r^{2}-Q^{2}\right)}\left(\frac{1}{r^{2}}-1\right) \\
& >-\frac{2 r_{0}^{2}}{r\left(1-Q^{2}\right)}, \quad r \in I_{0} .
\end{aligned}
$$

Integrating (57) and applying the condition $W(1)=1$, we obtain

$$
\begin{aligned}
W(r) & >1-\frac{2 r_{0}}{1-Q^{2}} \ln r_{0} \\
& =1-\frac{2 \sqrt{-Q \kappa}}{1-Q^{2}} \ln (-4 Q \kappa), \quad r \in I_{0} .
\end{aligned}
$$

It is clear that when $Q$ is close to $-\frac{1}{4 \kappa}$, the right-hand side of (58) stays positive. This proves that the set

$$
B^{+}=\left\{Q \in\left(-1,-\frac{1}{4 \kappa}\right) \mid W(r)>0 \quad \text { for all } r \in I_{0}\right\}
$$

where $W$ solves the initial-value problem

$W$ satisfies (47) and the initial condition $W(1)=1$,

$$
W^{\prime}(1)=b(Q),
$$

is nonempty. The continuous dependence of the solution on the initial condition and the parameters in the differential equation then indicates that $B^{+}$is open.
Similarly, it may be shown that the set

$$
B^{-}=\left\{Q \in\left(-1,-\frac{1}{4 \kappa}\right) \mid W(r)<0 \quad \text { for somel } r \in I_{0}\right\}
$$

is nonempty, where $W$ solves the initial-value problem (59). To see this, we may choose $Q$ to be close to -1 , which makes $W$ start with a sufficiently negative slope in view of (53) and drives $W$ to the negative value range quickly. Of course, $B^{-}$is also open.

By the connectedness of the interval $\left(-1,-\frac{1}{4 \kappa}\right)$, we see that

$$
B^{0}=\left(-1,-\frac{1}{4 \kappa}\right) \backslash\left(B^{+} \cup B^{-}\right) \neq \emptyset
$$

For $Q \in B^{0}$, we see that $W(r) \geq 0$ for all $r \in I_{0}$. Since $W^{\prime}(r)<0$ for $r \in I_{0}$, we must have $W\left(r_{0}\right)=0$.

In summary, we have obtained some $Q \in\left(-1,-\frac{1}{4 \kappa}\right)$ so that the solution of (59) satisfies

$$
W(r)>0 \quad \text { for } r_{0} \in\left[1, r_{0}\right), \quad W\left(r_{0}\right)=0,
$$$$
W^{\prime}(r)<0, \quad r \in\left[1, r_{0}\right] .
$$

Let $W$ be a solution of (59) satisfying (60). Then $W(r)<0$ for $r>r_{0}$, but $r$ is close to $r_{0}$, since $W\left(r_{0}\right)=0$ and $W^{\prime}\left(r_{0}\right)<0$. For $r>r_{0}$, we rewrite (52) as

$W^{\prime}(r)=\frac{4 r^{3}}{\left(r^{2}-1\right)\left(r^{2}-Q^{2}\right)}\left(\int_{1}^{r_{0}}+\int_{r_{0}}^{r}\right) \frac{1}{\rho}\left(1-\frac{r_{0}^{2}}{\rho^{2}}\right) W(\rho) d \rho$,

$$
r>r_{0} .
$$

We see that $W^{\prime}(r)$ for all $r>r_{0}$ in the interval of existence, since $W(r)<0$ beyond $r_{0}$. In particular, we have established that the set

$$
\begin{aligned}
\mathcal{Q}^{-} & =\left\{Q \in(-1,0] \mid W^{\prime}(r)<0\right. \text { for all } \\
r & >1 \text { and } W(r)<0 \text { for some } r>1\}
\end{aligned}
$$

is not empty. This set is readily seen to be open.

To proceed further, we define

$$
\mathcal{Q}^{+}=\left\{Q \in(-1,0] \mid W^{\prime}(r)>0 \text { for some } r>1\right\} .
$$

Then $\mathcal{Q}^{+} \neq \emptyset$. In fact, we may formally rewrite the local solution of (47) in the form (52) and use l'Hôpital's rule to find $W^{\prime}(1)$ again as before. In particular, for $Q<0$ and close to $Q=0$, we have $W^{\prime}(1)>0$. Thus, $W^{\prime}(r)>0$ for $r$ near $r=1$ and $r>1$. It is obvious that $\mathcal{Q}^{+}$is also open.

Now the connectedness of $(-1,0]$ shows that

$$
\mathcal{Q}^{0}=(-1,0] \backslash\left(\mathcal{Q}^{+} \cup \mathcal{Q}^{-}\right) \neq \emptyset \text {. }
$$


Take $Q \in \mathcal{Q}^{0}$. Then $Q<0$. For such $Q$, let $W$ be the corresponding solution of (59) where we no longer require $Q$ to be confined within the interval $\left(-1,-\frac{1}{4 \kappa}\right)$ but within $(-1,0)$. For such $W$, we have the properties

$$
W^{\prime}(r) \leq 0, \quad W(r) \geq 0, \quad r>1 .
$$

It is clear that in fact $W(r)>0$ for all $r>1$. Indeed, if there is some $r_{1}>1$ such that $W\left(r_{1}\right)=0$, then $W$ attains its interior minimum at $r_{1}$ such that $W^{\prime}\left(r_{1}\right)=0$. Applying the uniqueness theorem of the initial-value problem of ordinary differential equations, we derive $W \equiv 0$, which is false.

We claim $W^{\prime}(r)<0$ for all $r>1$. In fact, since $W(r)>0$, we have $W^{\prime}(r)<0$ for $r \in I_{0}$ in view of (52). Assume otherwise that $W^{\prime}\left(r_{1}\right)=0$ at some $r_{1}>r_{0}$ and that $r_{1}$ is the leftmost such point. Then (52) gives us

$$
\int_{1}^{r_{1}} \frac{1}{\rho}\left(1+\frac{4 Q \kappa}{\rho^{2}}\right) W(\rho) d \rho=0 .
$$

Hence, we have

$$
\begin{aligned}
W^{\prime}(r) & =\frac{4 r^{3}}{\left(r^{2}-1\right)\left(r^{2}-Q^{2}\right)}\left(\int_{1}^{r_{1}}+\int_{r_{1}}^{r}\right) \frac{1}{\rho}\left(1-\frac{r_{0}^{2}}{\rho^{2}}\right) W(\rho) d \rho \\
& =\frac{4 r^{3}}{\left(r^{2}-1\right)\left(r^{2}-Q^{2}\right)} \int_{r_{1}}^{r} \frac{1}{\rho}\left(1-\frac{r_{0}^{2}}{\rho^{2}}\right) W(\rho) d \rho>0, r>r_{1},
\end{aligned}
$$

since $W(r)>0$. This is false. Therefore, we arrive at the following slightly strengthened version of (61):

$$
W^{\prime}(r)<0, \quad W(r)>0, \quad r>1 .
$$

With the properties of $W$ established, we see in particular that

$$
W_{\infty} \equiv \lim _{r \rightarrow \infty} W(r)
$$

exists and $W_{\infty}[0,1)$. We further claim $W_{\infty}=0$. Otherwise, if $W_{\infty}>0$, then we have

$$
0<W_{\infty}<W(r)<1, \quad r>1 .
$$

Inserting this into (52), we see that there are constants $C_{0}, C_{1}, C_{2}>0$ such that

$$
W^{\prime}(r)>\frac{C_{0}}{r}\left(C_{1} \ln r-C_{2}\right), \quad r>r_{2}
$$

where $r_{2}>1$ is sufficiently large. In particular, $W^{\prime}(r)>0$ when $r$ is large enough, which is false again.

In summary, we have shown the existence of a solution $W$ of (47) which enjoys the properties

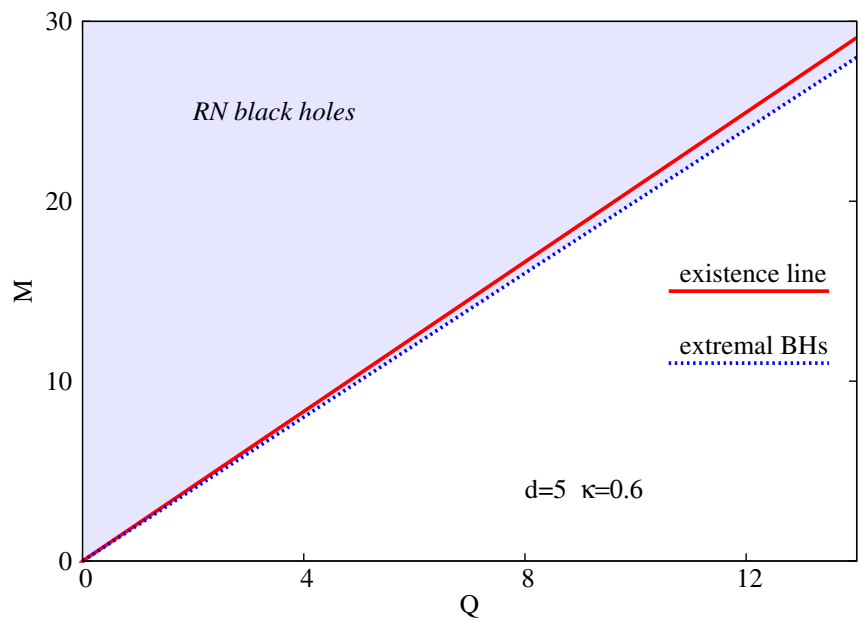

FIG. 5. Mass $M$ vs charge $Q$ for RN black holes in $d=5$ dimensions. The dotted blue curve corresponds to extremal $\mathrm{BHs}$ [RN BHs exist above it (shaded region)]. The non-Abelian clouds exists along the red line.

$$
\begin{aligned}
W(1) & =1 ; \quad W^{\prime}(r)<0, \quad W(r)>0, \\
1 & <r<\infty ; \quad W(\infty)=0,
\end{aligned}
$$

provided that $Q \in(-1,0)$ is suitably chosen and $\kappa$ stays in the interval $\left(\frac{1}{4}, \frac{1}{2}\right)$.

\section{The numerical results}

The smooth solution interpolating between (49) and (50) is constructed again by numerical integration. Not completely unexpected, the picture here is similar to that found for the model in Sec. III. In Fig. 5 we show the existence line found for $\kappa=0.6$. The profile of a typical solution of (47) is similar to that in Fig. 3 and we shall not display here.

As discussed in Refs. [31,32], these nA clouds may be continued past the point where they are an infinitesimally small perturbation of RNBHs. This results in a set of black holes with magnetic nA hair, which possess also a finite mass and electric charge. Remarkably, some of these nA configurations are stable under linear, spherically symmetric perturbations.

\section{CONCLUSIONS}

One of the most interesting developments in the physics of AdS spacetime was the discovery in 2008 by Gubser of the $p$-wave "holographic superconductors." The starting point there was the observation that RNAdS BH becomes unstable when considered as a solution of the EinsteinYang-Mills theory, with the appearance of a magnetic nA cloud close to the horizon. This feature occurs for a particular set of RNAdS configurations which form a line in the parameter space, the hairy BHs being the nonlinear realization of those marginally stable solutions.

The main purpose of this work was to show that, despite the different asymptotic structure of spacetime and the different horizon topology, the asymptotically flat RN black 
holes can also become unstable in the presence of nA gauge fields, with the occurrence of nA clouds around the horizon. We provided two examples of this, for $d=4$ and $d=5$ spacetime dimensions, in Secs. III and IV, respectively.

In contrast to the case of an AdS background, where one finds EYM solutions supporting the electric component of the nA $S U(2)$ connection $A_{t}$ in $d=4$ spacetime, the existence of such nA hairy solutions in the case of a flat background requires two crucial ingredients. The first one is that the gauge group should be large enough [at least $S O(d+1)$ for our models, with $d$ the spacetime dimension]. The second ingredient is the presence of some nonlinear terms in the $\mathrm{nA}$ connection and curvature, of higher order than the usual (quadratic) $F^{2}$ one. The higherorder terms used here are variously the (quartic) $F^{4}$ and the Chern-Simons terms. In both these two (flat background) cases, analytic proofs for the existence of the perturbed solutions were given in addition to numerical construction.

The general mechanism appears to be the following: in all cases, the RN BH remains a(n embedded Abelian) solution of the full model. However, for some range of the parameters, the extra terms mentioned above give a tachyonic mass for the vacuum perturbations of the nA magnetic fields around the Abelian solutions, with the appearance of a nA condensate. Similar to the AdS case, this implies the occurrence of a branch of fully nA BHs, which are generically thermodynamically favored over the Abelian configurations.

It would be interesting to further pursue the unveiled similarity of the Minkowskian case here with the AdS case, and to investigate the possible relevance of these aspects in providing analogies to phenomena observed in condensed matter physics.

\section{ACKNOWLEDGMENTS}

E. R. gratefully acknowledges funding from the FCT-IF program. This work was partially supported by the H2020MSCA-RISE-2015 Grant No. StronGrHEP-690904, and by the CIDMA project No. UID/MAT/04106/2013

\section{APPENDIX: THE NONLINEAR PROBLEMS}

\section{The EYM-AdS system in $\boldsymbol{d}=\mathbf{4}$ dimensions}

We consider a general class of EYM-AdS solutions with a line element

$$
\begin{aligned}
d s^{2} & =\frac{d r^{2}}{N(r)}+r^{2}\left(d \theta^{2}+f_{k}^{2}(\theta) d \varphi^{2}\right)-N(r) \sigma^{2}(r) d t^{2}, \\
N(r) & =k-\frac{2 m(r)}{r}+\frac{r^{2}}{L^{2}},
\end{aligned}
$$

where $k=0, \pm 1$ and

$$
f_{k}(\theta)= \begin{cases}\sin \theta, & \text { for } k=1 \\ \theta, & \text { for } k=0 \\ \sinh \theta, & \text { for } k=-1\end{cases}
$$

For any value of $k$, the metric (A1) possesses the same amount of symmetries, since $d \theta^{2}+f_{k}^{2}(\theta) d \varphi^{2}$ is the line element on a two-dimensional surface of constant curvature $2 k$. The spherically symmetric solutions have $k=1$, in which case one can find both solitons and black hole solutions [33]; for $k=0,-1$, one finds only "topological" black holes (see Ref. [34] for recent results on such configurations).

The $S U(2)$-YM Ansatz compatible with the symmetries of the line element (A1) has been proposed in Refs. [15,16], and reads

$$
\begin{aligned}
A= & \frac{1}{2}\left[V(r) \tau_{3} d t+w(r) \tau_{1} d \theta\right. \\
& \left.+\left(\frac{d f_{k}(\theta)}{d \theta} \tau_{3}+f_{k}(\theta) w(r) \tau_{2}\right) d \varphi\right]
\end{aligned}
$$

The gauge potentials $w(r), V(r)$ and the metric functions $m(r), \sigma(r)$ solve the EYM equations

$$
\begin{aligned}
& m^{\prime}=N w^{\prime 2}+\frac{r^{2} V^{\prime 2}}{2 \sigma^{2}}+\frac{\left(w^{2}-k\right)^{2}}{2 r^{2}}+\frac{w^{2} V^{2}}{N \sigma^{2}}, \\
& \sigma^{\prime}=\frac{2 \sigma}{r}\left(w^{\prime 2}+\frac{w^{2} V^{2}}{N^{2} \sigma^{2}}\right), \\
& w^{\prime \prime}+\left(\frac{N^{\prime}}{N}+\frac{\sigma^{\prime}}{\sigma}\right) w^{\prime}+\frac{w\left(k-w^{2}\right)}{r^{2} N}+\frac{w V^{2}}{N^{2} \sigma^{2}}=0, \\
& V^{\prime \prime}+\left(\frac{2}{r}-\frac{\sigma^{\prime}}{\sigma}\right) V^{\prime}-\frac{2 V}{r^{2} N} w^{2}=0 .
\end{aligned}
$$

One can easily see that $k=0$ is special, since the RN black hole with a nonzero electric field and a vanishing magnetic flux is a solution in that case only. For $k=1$, the magnetic field trivializes for $w(r)= \pm 1$. However, from the $w$ equation, this is not consistent with keeping a nonzero electric potential $V(r)$. Moreover, the solutions with $k=-1$ do not possess a vacuum, since one cannot find a real value of $w(r)$ which gives a vanishing magnetic field [even for $V(r) \equiv 0$ ].

\section{The EYM- $F^{4}$ system in $d$ spacetime dimensions}

We consider the following action in $d$ dimensions (with $\tau_{1}, \tau_{2}$ positive constants):

$$
S=\int d^{d} x \sqrt{-g}\left[\frac{1}{4} R-\frac{1}{2} \tau_{1} \operatorname{Tr}\left\{F(2)^{2}\right\}+\frac{3}{2} \tau_{2} \operatorname{Tr}\left\{F(4)^{2}\right\}\right],
$$

describing Einstein gravity coupled with the first two terms in the Yang-Mills hierarchy, ${ }^{4}$ considered in Ref. [35]. In the

\footnotetext{
${ }^{4} F(2 p)^{2}$ having been proposed as systems supporting instantons on $\mathbb{R}^{4 p}$ in Ref. [25].
} 
above relation, $F(2)=F_{\mu \nu}$ denotes the Yang-Mills curvature 2-form, while $F(4)=F_{\mu \nu \rho \sigma}$ is the Yang-Mills 4-form resulting from the total antisymmetrization of $F(2)$. The 4-form $F_{\mu \nu \rho \sigma}$ can be expressed conveniently as

$$
F_{\mu \nu \rho \sigma}=\left\{F_{\mu[\nu}, F_{\rho \sigma]}\right\},
$$

where $\left\{F_{\mu \nu}, F_{\rho \sigma}\right\}$ denotes the anticommutator of $F_{\mu \nu}$ and $F_{\rho \sigma}$, while the notation $F_{\mu[\nu} F_{\rho \sigma]}$ implies the cyclic symmetry on $(\nu, \rho, \sigma)$, i.e. $F_{\mu \nu} F_{\rho \sigma}+F_{\mu \rho} F_{\sigma \nu}+F_{\mu \sigma} F_{\nu \rho}$. This results in

$$
\operatorname{Tr}\left\{F(4)^{2}\right\}=6 \operatorname{Tr}\left[\left(F_{\mu \nu} F_{\rho \sigma}\right)^{2}-4\left(F_{\mu \rho} F_{\rho \nu}\right)^{2}+\left(F_{\mu \nu}^{2}\right)^{2}\right],
$$

which is analogous to the corresponding expression for the Gauss-Bonnet term in gravity. Unlike the latter, however, (A7) is not a total divergence and hence does not trivialize in $d=4$ dimensions, as long as the component $A_{0}$ of the YM connection is supported, which is achieved by choosing a large enough gauge group. In that case it can be expressed in the equivalent form displayed in Eq. (16).

A convenient parametrization of a spherically symmetric line element is

$$
\begin{aligned}
d s^{2} & =\frac{d r^{2}}{N(r)}+r^{2} d \Omega_{d-2}^{2}-N(r) \sigma^{2}(r) d t^{2}, \text { with } \\
N(r) & =1-\frac{2 m(r)}{r^{d-3}},
\end{aligned}
$$

with $m(r)$ the mass function and $d \Omega_{d-2}^{2}$ the metric on the $(d-2)$-sphere.

In $d$ spacetime dimensions, the minimal gauge group allowing a spherically symmetric nA ansatz containing both electric and magnetic parts is $S O(d+1)$. In what follows, we shall restrict the case to a consistent $S O(d-$ 1) $\times S O(2)$ truncation of the general ansatz, parametrized by two potentials, a magnetic one $w(r)$ and an electric one $V(r)$ :

$$
\begin{aligned}
A & =\frac{w(r)+1}{r} \gamma_{i j} \frac{x^{i}}{r} d x^{j}+V(r) \gamma_{d, d+1} d t, \quad \text { with } \\
i, j & =1, \ldots, d-1,
\end{aligned}
$$

$\gamma_{i j}$ being the representation matrices of $S O(d-1)$, and $\gamma_{d, d+1}$ those of the $S O(2)$, with subalgebras in $S O(d+1)$. The Cartesian coordinates $x^{i}$ are related to the spherical coordinates in (A8) as in flat space. The matrices $\gamma_{i}$ used here symbolize the $(d-1)$-dimensional Dirac gamma matrices $\Gamma_{i}$ when $d$ is odd, and the chiral matrices $\left(\Sigma_{i}, \tilde{\Sigma}_{i}\right)$ when $d$ is even.

A straightforward computation leads to the following equations of the model ${ }^{5}$ :

$$
\begin{aligned}
m^{\prime}= & r^{d-2}\left\{\tau_{1}\left[\frac{(d-2)}{2}\left(\frac{N w^{\prime 2}}{r^{2}}+\frac{1}{2}(d-3) W\right)+\frac{V^{\prime 2}}{2 \sigma^{2}}\right]+\tau_{2} W\left[\frac{V^{\prime 2}}{\sigma^{2}}+3(d-4)\left(\frac{N w^{\prime 2}}{r^{2}}+\frac{1}{4}(d-5) W\right)\right]\right\}, \\
\sigma^{\prime}= & \frac{\sigma}{r} w^{\prime 2}\left(\tau_{1}(d-2)+6 \tau_{2}(d-4) W\right), \quad w^{\prime \prime}\left(\tau_{1}+\frac{6(d-4)}{(d-2)} \tau_{2} W\right)+\tau_{1}\left[\left(\frac{N^{\prime}}{N}+\frac{\sigma^{\prime}}{\sigma}+\frac{d-4}{r}\right) w^{\prime}+\frac{(d-3)}{r^{2} N} w\left(1-w^{2}\right)\right] \\
& +\frac{\tau_{2}}{(d-2)}\left[\frac{6(d-4)}{r^{4}}\left(8 w\left(w^{2}-1\right) w^{\prime 2}+\left(\frac{N^{\prime}}{N}+\frac{\sigma^{\prime}}{\sigma}+\frac{d-8}{r}\right) r^{4} W w^{\prime}+\frac{(d-5)}{2 r^{2} N}\left(1-w^{2}\right)^{3}\right)\right. \\
& \left.+w\left(1-w^{2}\right) \frac{1}{r^{2}}\left(3(d-4)\left((d-5) W+\frac{4 N w^{\prime 2}}{r^{2}}\right)-\frac{4 V^{\prime 2}}{\sigma^{2}}\right)\right]=0, \\
& \left(V^{\prime} \frac{r^{d-2}}{\sigma}\left(\tau_{1}+\frac{2 \tau_{2}\left(1-w^{2}\right)^{2}}{r^{4}}\right)\right)^{\prime}=0 .
\end{aligned}
$$

The equations for the $S U(2) \times U(1)$ [i.e. $S O(3) \times S O(2)$ ] model introduced in Sec. III are found by taking $\tau_{1}=1, \tau_{2}=\tau$, $d=4$ in the above relations. Also, one can easily verify that the embedded Abelian RN BH in $d$ dimensions as given by

$$
m(r)=M-\frac{(d-3) Q^{2} \tau_{1}}{3 r^{d-3}}, \quad \sigma(r)=1, \quad w(r)= \pm 1, \quad V(r)=\frac{Q}{r^{d-3}}+c_{0},
$$

(with $M, Q$ being two constants fixing the mass and electric charge) is a solution.

\footnotetext{
${ }^{5}$ Note that, in order to simplify the relations, a factor of $d$ has been absorbed in the expression of $\tau_{1}, \tau_{2}$.
} 
Again, an infinitesimally small magnetic field is turned on by taking $w(r)=-1+\epsilon W(r)$. To leading order, the equation satisfied by $W(r)$ is

$$
\left(r^{d-4} N W^{\prime}\right)^{\prime}=\frac{2(d-3) W}{r^{6-d}}\left(\tau_{1}-\frac{4(d-3) \tau_{2}}{(d-2) r^{2}(d-3)}\right)
$$

\section{The EYM-CS system in $d=5$ dimensions}

We consider the following action:

$$
S=\int d^{5} x \sqrt{-g}\left[\frac{1}{4} R-\frac{1}{2} \operatorname{Tr}\left\{F_{\mu \nu} F^{\mu \nu}\right\}+\kappa \varepsilon^{\lambda \mu \nu \rho \sigma} \operatorname{Tr}\left\{A_{\lambda}\left(F_{\mu \nu} F_{\rho \sigma}-F_{\mu \nu} A_{\rho} A_{\sigma}+\frac{2}{5} A_{\mu} A_{\nu} A_{\rho} A_{\sigma}\right)\right\}\right] .
$$

The expression of a $d=5$ spherically symmetric line element reads

$$
d s^{2}=\frac{d r^{2}}{N(r)}+r^{2} d \Omega_{3}^{2}-N(r) \sigma^{2}(r) d t^{2}, \quad \text { with } \quad N(r)=1-\frac{m(r)}{r^{2}} .
$$

For the gauge group $S O(6)$, the corresponding spherically symmetric, time-independent YM ansatz is given by (43), in terms of a magnetic potential $w(r)$ and an electric one $V(r)$. Then the equations of the model are

$$
\begin{aligned}
m^{\prime} & =\frac{1}{2}\left(3 r\left(N w^{\prime 2}+\frac{\left(w^{2}-1\right)^{2}}{r^{2}}\right)+\frac{r^{3}}{\sigma^{2}} V^{\prime 2}\right), \quad \frac{\sigma^{\prime}}{\sigma}=\frac{3 w^{\prime 2}}{2 r}, \\
\left(r \sigma N w^{\prime}\right)^{\prime} & =\frac{2 \sigma w\left(w^{2}-1\right)}{r}+8 \kappa V^{\prime}\left(w^{2}-1\right), \quad\left(\frac{r^{3} V^{\prime}}{\sigma}\right)^{\prime}=24 \kappa\left(w^{2}-1\right) w^{\prime} .
\end{aligned}
$$

[1] R. Gregory and R. Laflamme, Black Strings and p-branes Are Unstable, Phys. Rev. Lett. 70, 2837 (1993).

[2] S. S. Gubser, On nonuniform black branes, Classical Quantum Gravity 19, 4825 (2002).

[3] O. J. C. Dias, P. Figueras, R. Monteiro, J. E. Santos, and R. Emparan, Instability and new phases of higher-dimensional rotating black holes, Phys. Rev. D 80, 111701 (2009); O. J. C. Dias, P. Figueras, R. Monteiro, and J. E. Santos, Ultraspinning instability of rotating black holes, Phys. Rev. D 82, 104025 (2010).

[4] J. C. Dias, J. E. Santos, and B. Way, Rings, ripples, and rotation: Connecting black holes to black rings, J. High Energy Phys. 07 (2014) 045; R. Emparan, P. Figueras, and M. Martinez, Bumpy black holes, J. High Energy Phys. 12 (2014) 072.

[5] J. Kunz and Y. Brihaye, New sphalerons in the WeinbergSalam theory, Phys. Lett. B 216, 353 (1989).

[6] L. G. Yaffe, Static solutions of $S U(2)$ Higgs theory, Phys. Rev. D 40, 3463 (1989).

[7] S. Hod, Stationary scalar clouds around rotating black holes, Phys. Rev. D 86, 104026 (2012); 86, 129902 (2012).
[8] C. A. R. Herdeiro and E. Radu, Kerr Black Holes with Scalar Hair, Phys. Rev. Lett. 112, 221101 (2014); Construction and physical properties of Kerr black holes with scalar hair, Classical Quantum Gravity 32, 144001 (2015).

[9] C. L. Benone, L. C. B. Crispino, C. Herdeiro, and E. Radu, Kerr-Newman scalar clouds, Phys. Rev. D 90, 104024 (2014).

[10] O. Chodosh and Y. Shlapentokh-Rothman, Time-periodic Einstein-Klein-Gordon bifurcations of Kerr, arXiv: 1510.08025 .

[11] S. S. Gubser, Breaking an Abelian gauge symmetry near a black hole horizon, Phys. Rev. D 78, 065034 (2008).

[12] S. S. Gubser, Colorful Horizons with Charge in Anti-de Sitter Space, Phys. Rev. Lett. 101, 191601 (2008).

[13] G. T. Horowitz, Introduction to holographic superconductors, Lect. Notes Phys. 828, 313 (2011).

[14] K. M. Lee, V. P. Nair, and E. J. Weinberg, A Classical Instability of Reissner-Nordstrom Solutions and the Fate of Magnetically Charged Black Holes, Phys. Rev. Lett. 68, 1100 (1992). 
[15] J. J. Van der Bij and E. Radu, New hairy black holes with negative cosmological constant, Phys. Lett. B 536, 107 (2002).

[16] R. B. Mann, E. Radu, and D. H. Tchrakian, Non-Abelian solutions in $\operatorname{AdS}(4)$ and $d=11$ supergravity, Phys. Rev. D 74, 064015 (2006).

[17] S. W. Hawking and G. F. R. Ellis, The Large Scale Structure of Space-Time, Cambridge Monographs on Mathematical Physics (Cambridge University Press, Cambridge, England, 1973).

[18] J. L. Friedman, K. Schleich, and D. M. Witt, Topological Censorship, Phys. Rev. Lett. 71, 1486 (1993); P. T. Chrusciel and R. M. Wald, On the topology of stationary black holes, Classical Quantum Gravity 11, L147 (1994).

[19] D. V. Galtsov and A. A. Ershov, Non-Abelian baldness of colored black holes, Phys. Lett. A 138, 160 (1989); P. Bizon and O. T. Popp, No hair theorem for spherical monopoles and dyons in $S U(2)$ Einstein Yang-Mills Theory, Classical Quantum Gravity 9, 193 (1992).

[20] D. V. Galtsov and M. S. Volkov, Charged non-Abelian $S U$ (3) Einstein Yang-Mills black holes, Phys. Lett. B 274, 173 (1992).

[21] M.S. Volkov and D. V. Galtsov, Non-Abelian Einstein Yang-Mills black holes, JETP Lett. 50, 346 (1989); H. P. Kuenzle and A. K. Masood-ul-Alam, Spherically symmetric static $S U(2)$ Einstein Yang-Mills fields, J. Math. Phys. (N.Y.) 31, 928 (1990); P. Bizon, Colored Black Holes, Phys. Rev. Lett. 64, 2844 (1990).

[22] E. Radu and D. H. Tchrakian, Stable black hole solutions with non-Abelian fields, Phys. Rev. D 85, 084022 (2012).

[23] B. Zwiebach, Curvature squared terms and string theories, Phys. Lett. 156B, 315 (1985).

[24] T. H. R. Skyrme, A unified field theory of mesons and baryons, Nucl. Phys. 31, 556 (1962).

[25] D. H. Tchrakian, Spherically symmetric gauge field configurations with finite action in $4 p$ dimensions ( $p=$ integer), Phys. Lett. 150B, 360 (1985).

[26] R. Manvelyan, E. Radu, and D. H. Tchrakian, New AdS non-Abelian black holes with superconducting horizons, Phys. Lett. B 677, 79 (2009).
[27] V. Paturyan, E. Radu, and D. H. Tchrakian, ReissnerNordström black holes with non-Abelian hair (to be published).

[28] A. Chakrabarti and D. H. Tchrakian, Gravitation with superposed Gauss-Bonnet terms in higher dimensions: Black hole metrics and maximal extensions, Phys. Rev. D 65, 024029 (2001).

[29] M. Gunaydin, L. J. Romans, and N. P. Warner, Compact and noncompact gauged supergravity theories in fivedimensions, Nucl. Phys. B272, 598 (1986).

[30] M. Cvetic, H. Lu, C. N. Pope, A. Sadrzadeh, and T. A. Tran, Consistent $S O(6)$ reduction of type IIB supergravity on S(5), Nucl. Phys. B586, 275 (2000).

[31] Y. Brihaye, E. Radu, and D. H. Tchrakian, Asymptotically Flat, Stable Black Hole Solutions in Einstein-Yang-MillsChern-Simons Theory, Phys. Rev. Lett. 106, 071101 (2011).

[32] Y. Brihaye, E. Radu, and D. H. Tchrakian, Einstein-YangMills-Chern-Simons solutions in $D=2 n+1$ dimensions, Phys. Rev. D 84, 064015 (2011).

[33] E. Winstanley, Existence of stable hairy black holes in $S U$ (2) Einstein Yang-Mills theory with a negative cosmological constant, Classical Quantum Gravity 16, 1963 (1999); J. Bjoraker and Y. Hosotani, Stable Monopole and Dyon Solutions in the Einstein-Yang-Mills Theory in Asymptotically Anti-de Sitter Space, Phys. Rev. Lett. 84, 1853 (2000); Monopoles, dyons and black holes in the fourdimensional Einstein-Yang-Mills theory, Phys. Rev. D 62, 043513 (2000).

[34] J.E. Baxter, Existence of topological hairy dyons and dyonic black holes in anti-de Sitter $S U(N)$ EinsteinYang-Mills theory, J. Math. Phys. (N.Y.) 57, 022505 (2016); B. C. Nolan and E. Winstanley, On the stability of dyons and dyonic black holes in Einstein-Yang-Mills theory, Classical Quantum Gravity 33, 045003 (2016); E. Winstanley, A menagerie of hairy black holes, arXiv: 1510.01669.

[35] Y. Brihaye, A. Chakrabarti, and D. H. Tchrakian, Particlelike solutions to higher order curvature Einstein-Yang-Mills systems in $d$-dimensions, Classical Quantum Gravity 20, 2765 (2003). 